\title{
Potential biomarker of metformin action
}

\author{
Ling He', Shumei Meng', Emily L Germain-Lee ${ }^{2,3}$, Sally Radovick ${ }^{2}$ and \\ Fredric E Wondisford ${ }^{1}$
}

${ }^{1}$ Division of Metabolism and ${ }^{2}$ Division of Endocrinology, Departments of Pediatrics, Physiology and Medicine, Johns Hopkins University School of Medicine, 600 North Wolfe Street, Baltimore, Maryland 21287, USA

${ }^{3}$ Kennedy Krieger Institute, Baltimore, Maryland 21287, USA
Correspondence should be addressed to L He or F E Wondisford Emails heling@jhmi.edu or fwondis1@jhmi.edu

\begin{abstract}
Metformin is a first-line, anti-diabetic agent prescribed to over 150 million people worldwide. The main effect of metformin is to suppress glucose production in the liver; however, there is no reliable biomarker to assess the effectiveness of metformin administration. Our previous studies have shown that phosphorylation of CBP at S436 is important for the regulation of hepatic glucose production by metformin. In current study, we found that CBP could be phosphorylated in white blood cells (WBCs), and CBP phosphorylation in the liver and in WBCs of mice had a similar pattern of change during a fasting time course experiment. These data suggests that CBP phosphorylation in WBCs may be used as a biomarker of metformin action in the liver.
\end{abstract}
Key Words
- diabetes
- glucose metabolism
- liver
- metabolism

\section{Introduction}

Diabetes mellitus is increasing in prevalence worldwide due to dietary excesses and sedentary lifestyles. About 25.8 million individuals in the USA have diabetes, accounting for $8.3 \%$ of the population. Type 2 diabetes previously was limited to Western developed nations but now has spread worldwide. Data from the International Diabetes Federation (http://www.idf.org/worlddiabetesday/toolkit/gp/ facts-figures) show that diabetes affects at least 371 million individuals worldwide. Diabetes mellitus significantly increases the morbidity and mortality of those affected, resulting in substantial direct and indirect costs to society. As of 2012, 4.8 million people died, half under the age of 60 , and 471 billion USD were spent on the care of diabetes.

The primary treatment goal of patients with diabetes is to control their hyperglycemia so as to reduce the occurrence of diabetes-related complications. Metformin is a first-line anti-diabetic agent and is now the most widely prescribed drug for the treatment of diabetes.
Metformin or dimethylbiguanide is a derivative of guanidine, and compounds related to guanidine are the active ingredients in Galega officinalis, a plant that was used in folklore medicine to treat symptoms of diabetes in medieval Europe (Bailey \& Day 1989). In the 1920s, several biguanides including metformin and phenformin were synthesized and have been used to treat diabetes since the 1950s. However, phenformin was discontinued from the pharmaceutical use in most countries by the end of the 1970s due to its association with lactic acidosis (Witters 2001). Now, over 150 million people worldwide take metformin. Recently, this drug has received extra attention because many studies have suggested that diabetic patients treated with metformin exhibit a reduction in cancer incidence (Evans et al. 2005, Libby et al. 2009, Landman et al. 2010). The main effect of metformin is to suppress glucose production in the liver (Hundal et al. 2002, Takashima et al. 2010), and it has been

Published by Bioscientifica Ltd 
proposed that metformin suppresses hepatic glucose production (HGP) through the LKB1-AMPK pathway (Zhou et al. 2001, Shaw et al. 2005).

We and other investigators have shown that the CREB co-activators CBP and p300 are important in the regulation of HGP (Zhou et al. 2004, Liu et al. 2008, He et al. 2009, 2012). CBP and p300 are highly related proteins and share extensive homology at the amino acid level $(\sim 60 \%)$ and harbor several conserved domains such as the histidine-rich domains (CH1-3), the CREB-binding domain (KIX domain), the bromodomain, the histone acetyltransferase (HAT) domain, and the steroid receptor co-activator interacting (SID) domain (Goodman \& Smolik 2000). Through these domains, CBP and p300 function as scaffold proteins, interacting simultaneously with a variety of transcription factors and the basal transcription machinery. We have previously reported that the phosphorylation of CBP at S436 by metformin leads to the disassembly of CREB-CBP-CRTC2 complex and the suppression of glucose production in the liver (He et al. 2009). Despite knowing the cellular mechanism of metformin action, no reliable clinical markers of metformin action or toxicity, beyond those found through empiric dosing regimens, are available. We, therefore, tested whether the phosphorylation of CBP in white blood cells (WBCs) can be used as surrogate biomarker of metformin action.

\section{Materials and methods}

\section{Isolation of WBCs from mice}

All animal protocols were approved by the Institutional Animal Care and Use Committee of the Johns Hopkins University. After anesthesia with isoflurane, blood samples were collected from the heart and immediately transferred into EDTA treated tubes. The blood samples were then mixed with isolation buffer $\left(0.1 \%\right.$ glucose, $5 \mu \mathrm{M} \mathrm{CaCl}_{2}$, $98 \mu \mathrm{M} \mathrm{MgCl}_{2}, 0.54 \mathrm{mM} \mathrm{KCl}, 14.5 \mathrm{mM}$ Tris, $\mathrm{pH}$ 7.6, and $0.126 \mathrm{M} \mathrm{NaCl}$ ). The diluted blood samples were gently added on the top of Ficoll-Pague plus (GE Health, Pittsburg, PA, USA), and subjected to centrifugation at $300 \mathrm{~g}$ for $40 \mathrm{~min}$. The medium buffy coat layer was collected and washed with isolation buffer twice.

\section{Metformin tolerance test}

P300 knockin and littermate control mice were fed a high-fat diet ( $60 \%$ of calorie) for 4 weeks. Mice were fasted for $4 \mathrm{~h}$ followed by an i.p. injection of $250 \mathrm{mg} / \mathrm{kg}$ metformin, and blood glucose levels were measured at indicated time points (He et al. 2012).

\section{Glucose production assay}

Mouse primary hepatocytes from WT and CBPS436A mutant mice were cultured in William's medium $\mathrm{E}$ supplemented with ITS (insulin, transferrin, selenium; BD Biosciences, San Jose, CA, USA) and dexamethasone. At $16 \mathrm{~h}$ after planting, primary hepatocytes were subjected to $3 \mathrm{~h}$ serum starvation. The cells were washed twice with PBS and the medium was replaced with $1 \mathrm{ml}$ of glucose production medium consisting of glucose-free DMEM supplemented with $20 \mathrm{mM}$ sodium lactate (Sigma) and $2 \mathrm{mM}$ sodium pyruvate (Sigma) or with metformin (Enzo Life Sciences, Farmingdale, NY, USA) and $0.2 \mathrm{mM}$ Bt-cAMP (Sigma). After $6 \mathrm{~h}$ incubation, both the medium and cells were collected. The medium was used to determine glucose concentrations with EnzyChrom Glucose Assay Kit (He et al. 2012, 2013).

\section{Metformin treatment of human subjects}

Two obese female subjects (BMI $Z$-scores $>2.5$ ) were evaluated in the Institute for Clinical and Translational Research at the Johns Hopkins Hospital (Baltimore, MD, USA). This study was approved by the Institutional Review Board of the Johns Hopkins Medical Institutions. Informed consent was obtained from the adult participant (subject no. 1, age 27 years) and from the parents of the child participant (subject no. 2, age 15 years). Before the initiation of metformin, oral glucose tolerance tests were carried out on both subjects as part of their routine clinical care, and both subjects demonstrated glucose intolerance $(120 \mathrm{~min}$ serum glucose levels of 163 and $156 \mathrm{mg} / \mathrm{dl}$ respectively and HbA1c levels of 6.0 and 5.9\% respectively). Both subjects were metformin-naïve and underwent a $12 \mathrm{~h}$ overnight fast after which blood samples were collected into $\mathrm{K}_{2}$ EDTA-coated tubes before initiating therapy with a single dose of $500 \mathrm{mg}$ metformin. Samples were again obtained at $30 \mathrm{~min}$ and WBCs were isolated according to the protocol described earlier.

\section{Immunoblot}

Immunoblot analysis was conducted as previously described (He et al. 2009, 2012). Cellular lysates were sonicated for $15 \mathrm{~s}$ three times sequentially, western immunoblot analysis was carried out to examine the target proteins with antibodies against CBP and phospho-CBP.

Published by Bioscientifica Ltd. 


\section{Statistical analyses}

Statistical significance was calculated with a Student's $t$-test and ANOVA test. Significance was accepted at the level of $P<0.05$.

\section{Results}

\section{Phosphorylation of CBP by insulin and metformin suppresses glucose production}

The main effect of metformin is to suppress glucose production in the liver (Hundal et al. 2002), and metformin administration reduces HGP by over $60 \%$ in a euglycemicclamp study (Takashima et al. 2010). The suppression of HGP by metformin is through activation of the AMPK pathway, which subsequently inhibits gluconeogenic gene expression (Shaw et al. 2005, He et al. 2009, Takashima et al. 2010). The phosphorylation of CBP at Ser 436 by insulin and metformin disassembles the CREB-CBP-CRTC2 complex and suppresses HGP (Fig. 1A; He et al. 2009). Importantly, low metformin concentrations found in the portal vein of animals $(60-80 \mu \mathrm{M})$ are able to mediate phosphorylation of CBP in Hepa1-6 cells (Fig. 1B; Wilcock \& Bailey 1994). We have previously reported that a germline mutation of this CBP phosphorylation site (S436A) in mice leads to inappropriate activation of gluconeogenesis and metformin resistance (Zhou et al. 2004, He et al. 2012). Primary hepatocytes from CBPS436A mutant mouse produced significantly more glucose in basal condition and after cAMP stimulation than hepatocytes from WT littermate mice (Fig. 2A), and metformin had less effect on the suppression of cAMP-stimulated glucose production the in hepatocytes from CBPS436A mutant mouse. Interestingly, this phosphorylation site does not exist in its closely related protein, p300. Therefore, p300 binds to CREs containing gluconeogenic gene and constitutively maintains basal gluconeogenesis in the liver (He et al. 2012). In a phosphorylation-competent p300G442S knockin mouse model that bears a reconstituted phosphorylation site found in CBP, we found exaggerated hypoglycemia in a metformin tolerance test (Fig. 2B). These data substantiate further the importance of CBP phosphorylation in mediating the suppression of HGP by metformin.

\section{CBP phosphorylation in liver and in WBCs of mice}

Our previous studies showed that insulin mediated the phosphorylation of CBP at S436 in the liver (Zhou et al. 2004, He et al. 2009), whereas activation of the cAMP-PKA
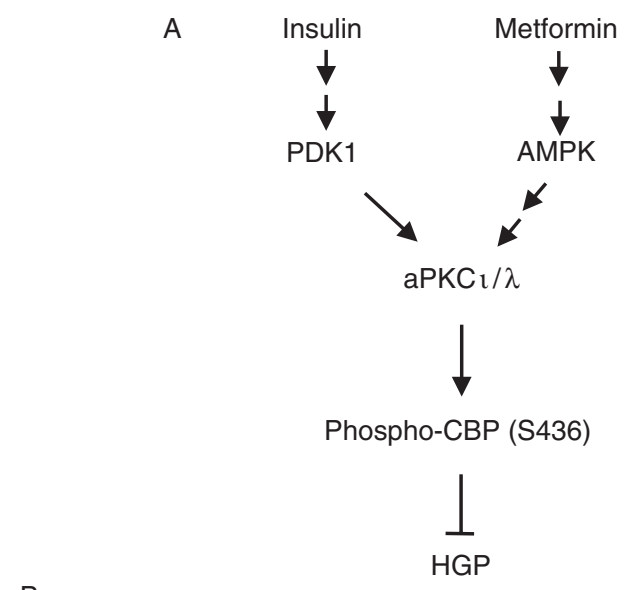

B
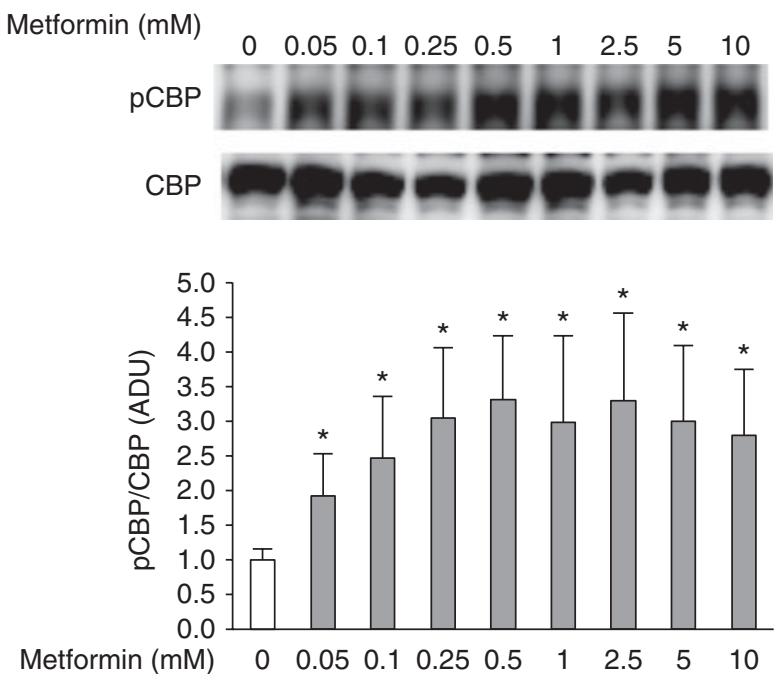

Figure 1

A proposed model for the phosphorylation of CBP by insulin and metformin (A). Hepa1-6 cells were treated with indicated amount of metformin for $8 \mathrm{~h}$ before the harvesting (B, upper panel), and immunoblotted with antibodies of CBP and phospho-CBP. Each lane represents sample pooled from three treatments. Densitometric analysis of the protein levels (pCBP/CBP), $n=3$ (B, lower panel). Asterisk (*) indicates that mean levels of the metformin-treatment group and the control group differed $(P<0.05)$. ADU, arbitrary densitometric units.

pathway antagonized CBP phosphorylation by insulin (He et al. 2009). These results suggest that CBP phosphorylation status might change in fasted and fed states in the liver. Indeed, CBP phosphorylation in the liver increased dramatically in the fed state when compared with the fasted state (Zhou et al. 2004, He et al. 2009). To investigate further whether feeding will increase hepatic CBP phosphorylation in fasted mice, we conducted a re-feeding time course experiment. In this experiment, mice were first fasted for $18 \mathrm{~h}$ and then given access to food and killed at different points during

Published by Bioscientifica Ltd 
A
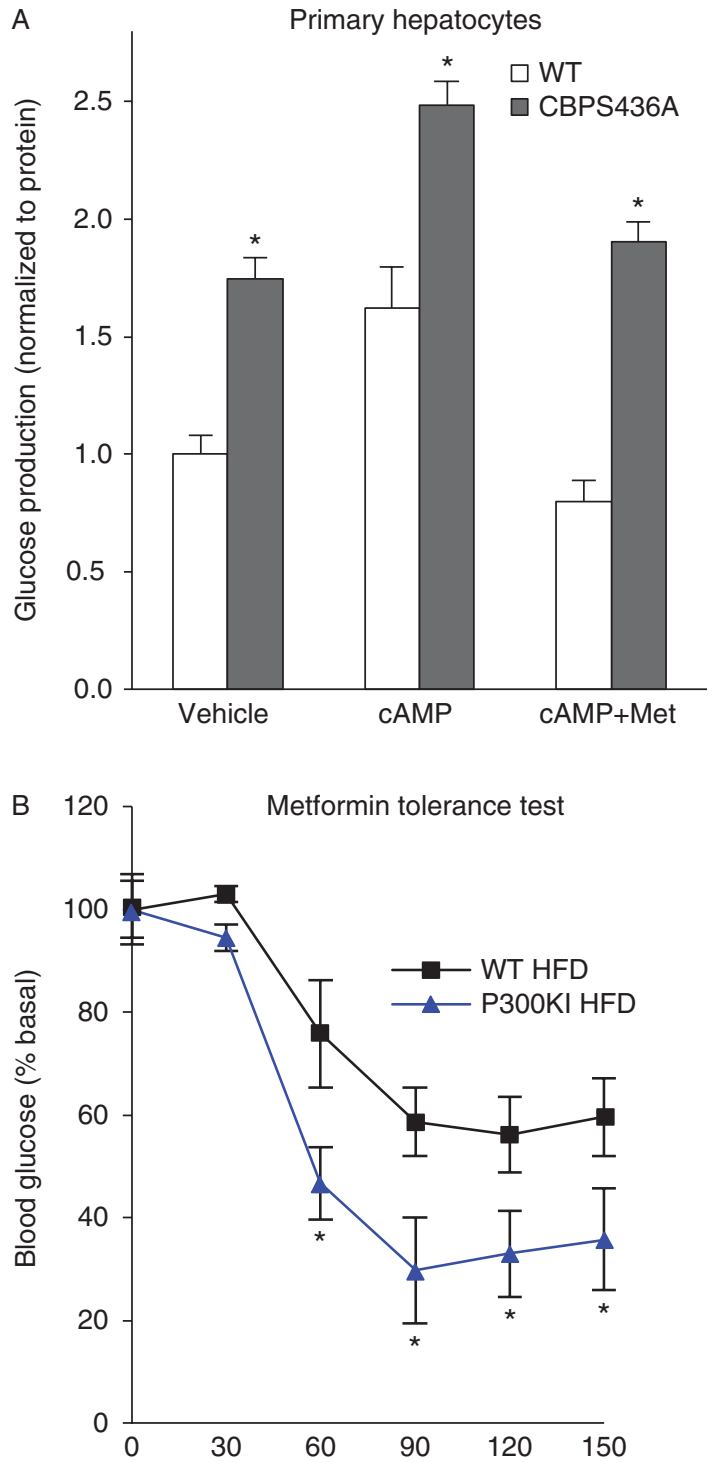

Figure 2

Metformin mediated CBP phosphorylation at S436. Primary hepatocytes from CBPS436A knockin mouse produced more glucose than that of hepatocytes from control mouse in the presence or absence CAMP treatment. After $3 \mathrm{~h}$ of serum starvation, cells were washed with PBS twice, and then $1 \mathrm{mM}$ metformin and $0.2 \mathrm{mM}$ cAMP were added in glucose production medium for $6 \mathrm{~h}(\mathrm{~A})$. After 4 weeks of feeding on a high-fat diet, p300 knockin and littermate control mice were subjected to fasting for $4 \mathrm{~h}$, and then mice received $250 \mathrm{mg} / \mathrm{kg}$ of metformin through i.p. injection. Blood glucose levels were measured at indicated time points (B). Asterisk $\left.{ }^{*}\right)$ signifies that groups with same treatment are significantly different $(P<0.05)$

an $8 \mathrm{~h}$ time period. After long-term fasting, re-feeding led to rapid hepatic CBP phosphorylation, which was maintained during the re-feeding period and peaked after $3 \mathrm{~h}$ of re-feeding (Fig. 3).

Metformin is now increasingly being used to treat children with type 2 diabetes mellitus and obesity due to its efficacy in adult patients. However, no reliable clinical markers of metformin action or toxicity, beyond those found through empiric dosing regimens, are available. In order to identify an applicable biomarker for metformin action in the liver, we monitored the phosphorylation levels of CBP in WBCs of mice in a fasting time course experiment. In this experiment, blood samples were collected at different time points. We found that CBP could be phosphorylated in WBCs in the fed state; however, CBP was dephosphorylated after $2 \mathrm{~h}$ of fasting accompanied by a decrease in blood glucose levels (Fig. 4A and B). Next, we conducted another fasting time course experiment to assess the CBP phosphorylation status in the liver. As shown in Fig. 4C, fasting led to the rapid dephosphorylation of CBP in the liver. Importantly, both the phosphorylation of CBP in WBC lysates and liver have a similar pattern, suggesting that CBP phosphorylation in WBCs can be used as a biomarker of metformin action in the liver.

\section{Metformin mediates CBP phosphorylation in liver and in WBCs from mice and humans}

Having seen CBP dephosphorylation in WBC lysates follows a similar pattern of change to that found in the
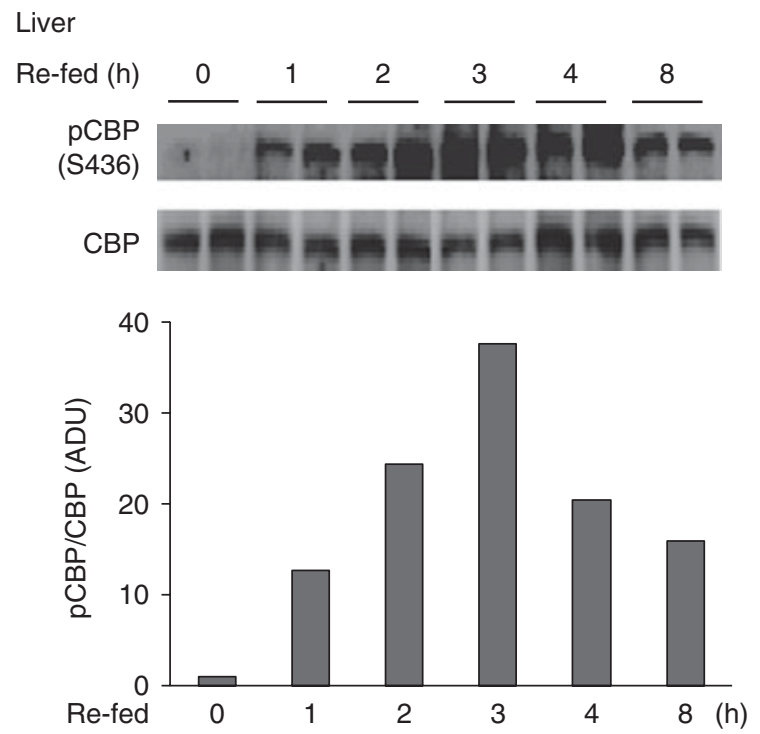

Figure 3

Re-feeding increased hepatic CBP phosphorylation. Mice were allowed to access food after $18 \mathrm{~h}$ of fasting and killed at indicated time points. Hepatic CBP phosphorylation levels were determined using anti phospho-CBP antibody, then, same blot was stripped and used to examine total CBP protein (upper panel). Lower panels depict densitometric analysis of phospho- $C B P(p C B P)$ relative to total $C B P$. 
A

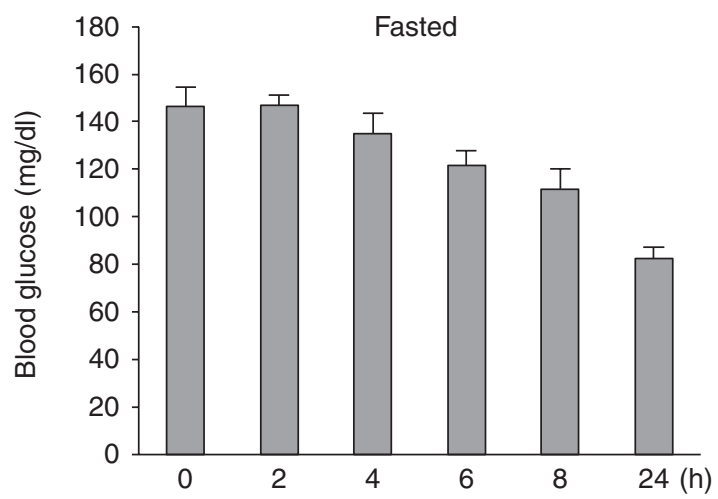

B

WBC
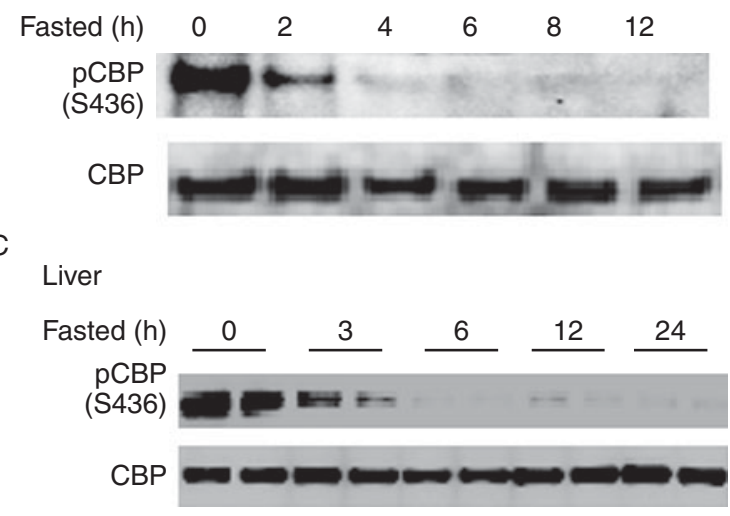

Figure 4

Fasting led to decreased CBP phosphorylation in liver and in WBC. Blood glucose levels of WT mice during a $24 \mathrm{~h}$ fasting period are shown $(n=4)(\mathrm{A})$. Phospho-CBP and total CBP in WBCs collected from fasted WT mice (B). Time course of changes in phosphorylated CBP and total CBP in liver of fasted mice (C).

liver during a fasting time course experiment (Fig. 4B and $\mathrm{C}$ ), we next probed whether metformin is able to mediate CBP phosphorylation in WBCs. Sixteen hours fasted mice were used because CBP is dephosphorylated during fasting. Mice received metformin through i.p. injection and blood samples and liver tissues were collected $15 \mathrm{~min}$ after the metformin administration. As shown in Fig. 5A, metformin treatment increased CBP phosphorylation both in the liver and in WBCs.

We investigated further whether metformin was able to mediate CBP phosphorylation in WBC lysates from humans. Two obese human subjects were given $500 \mathrm{mg}$ metformin orally after a $12 \mathrm{~h}$ fast, and blood samples were collected $30 \mathrm{~min}$ later. Metformin administration also increased CBP phosphorylation in WBC lysates in these two human subjects (Fig. 5B). Thus, CBP phosphorylation in WBCs may be useful as a biomarker for hepatic CBP
A

(Mouse)
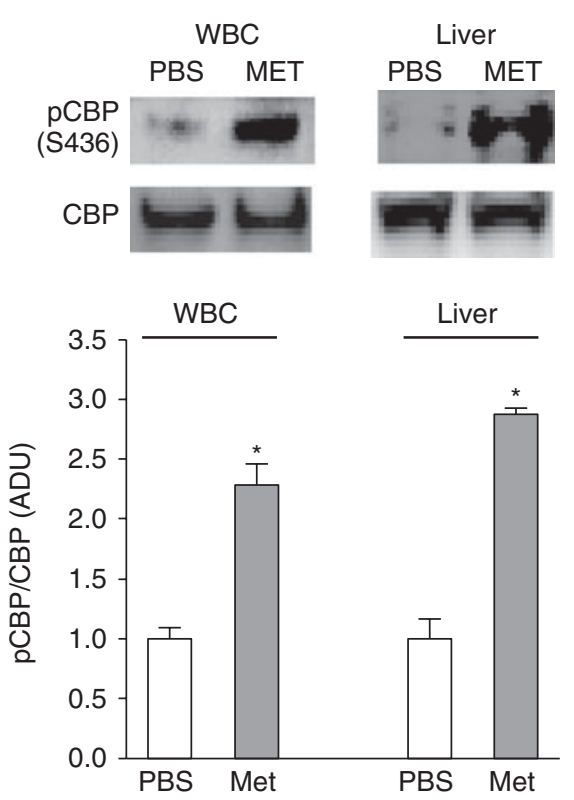

B

(Human)
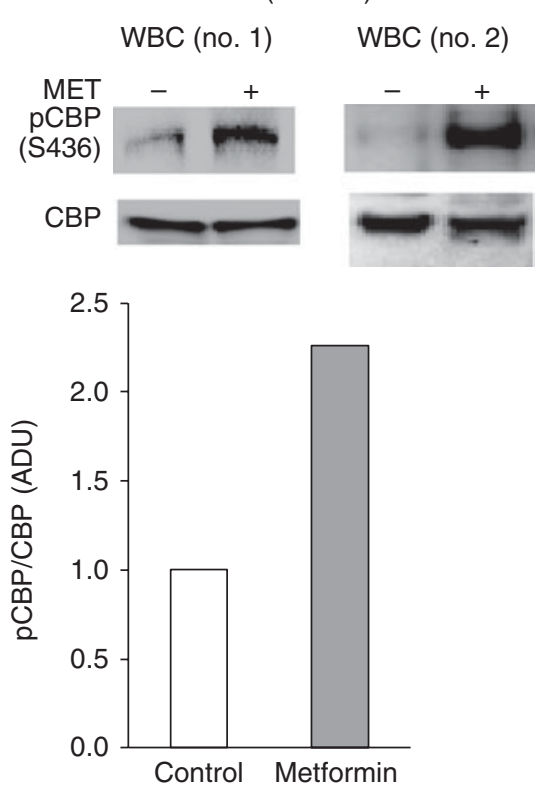

Figure 5

Metformin stimulated CBP phosphorylation in liver and in WBC of mice and human subjects. Phosphorylated CBP and total CBP in pooled liver or WBC samples from same WT mice (three mouse) with or without metformin treatment (15 $\mathrm{min}$ ) via i.p. injection ( $\mathrm{A}$, upper panel). Densitometric analysis of the protein levels (pCBP/CBP), $n=3$ (A, lower panel). In fasted obese human subjects with impaired glucose tolerance, phosphorylated CBP and total CBP in WBCs before and 30 min after administration of oral metformin $(500 \mathrm{mg})(B$, upper panel). Densitometric analysis of the protein levels ( $(\mathrm{C} C B \mathrm{~B} / \mathrm{CBP})$ from two human samples (B, lower panel). Asterisk (*) indicates that mean levels of the metformin-treatment group and the control group differed significantly $(P<0.05)$. 
phosphorylation and as a clinical biomarker for metformin effectiveness.

\section{Discussion}

The relentless increase in type 2 diabetes mellitus is a serious global public health emergency that affects the economies of all nations, particularly in developing countries. Metformin is a widely prescribed first-line, anti-diabetic drug and has been recognized as the most effective intervention for the treatment of type 2 diabetes mellitus due to its favorable safety profile and low cost. Recently, metformin has been promoted for a range of other indications: cancer prevention (Evans et al. 2005, Libby et al. 2009, Landman et al. 2010), improvement of the outcome of heart failure (Gundewar et al. 2009, Roussel et al. 2010, Aguilar et al. 2011), and protection against nonalcoholic fatty liver disease (Schwimmer et al. 2005, Nadeau et al. 2009). However, there is no reliable biomarker to assess metformin efficacy in the clinical setting. Our previous findings show that the phosphorylation of CBP at S436 is important for the regulation of HGP by insulin and metformin. Interestingly, this CBP phosphorylation site does not exist in its closely related p300 protein, suggesting a different role for p300 in glucose metabolism. The P300G422S knockin mouse model, containing a reconstituted phosphorylation site found in CBP, exhibited exaggerated hypoglycemia in a metformin tolerance test (Fig. 2B). In addition, the P300G422S knockin mouse model also displayed significantly lower blood glucose levels (He et al. 2012) and provides additional evidence for specificity of action in the $\mathrm{CBP} / \mathrm{p} 300$ family of co-activators. These data confirmed further the importance of CBP phosphorylation in the regulation of HGP. Moreover, activation of the cAMPPKA pathway negatively affected CBP phosphorylation (He et al. 2009), suggesting that elevation of glucagon levels, which is often seen in patients with diabetes, would decrease the efficacy of metformin in suppressing HGP.

Because phosphorylation of CBP is a biomarker of metformin action, a liver biopsy could be used to measure metformin efficacy. However, given the costs and risk of this procedure, this would be impractical. Based on this consideration, we tested CBP phosphorylation in WBC lysates and found that CBP was phosphorylated in WBCs in a similar pattern to CBP phosphorylation in the liver (Fig. 4). Metformin administration increased CBP phosphorylation in WBCs as well as in the liver of mice (Fig. 5A). Aside from these animal data, we also found that CBP could be phosphorylated by metformin in WBCs of two human obese subjects (Fig. 5B). Our study suggests, therefore, that CBP phosphorylation in WBCs can be used as a biomarker for hepatic CBP phosphorylation.

Hyperglycemia in diabetic patients is caused mainly by unregulated HGP, although decreased glucose uptake and utilization in peripheral tissues such as muscle and adipose tissue due to insulin resistance also contribute to the development of hyperglycemia (Guo 2014). As the main effect of metformin is to suppress glucose production in the liver (Hundal et al. 2002, Takashima et al. 2010), CBP phosphorylation in WBCs reflects hepatic CBP phosphorylation (Fig. 4) and the suppression of HGP (He et al. 2009); measurement of CBP phosphorylation in WBCs could be used to assess metformin efficacy. In some cases, high metformin doses are administered in order to control hyperglycemia, and such patients might be at higher risk for toxicity. However, a blood biomarker might reveal that lower metformin dosage can achieve the same effect on suppression of HGP. In addition, it remains unclear whether some patients are resistant to metformin and whether a biomarker would be essential to confirm this point. Although promising, these data must be validated in diabetic patients, who are metformin naive and in whom CBP phosphorylation, glucose tolerance, and insulin sensitivity are measured before and after metformin treatment.

\section{Declaration of interest}

The authors declare that there is no conflict of interest that could be perceived as prejudicing the impartiality of the research reported.

\section{Funding}

This work was supported in part by grants from the National Institute of Diabetes and Digestive and Kidney Diseases: R00DK085142 (L H), R01 DK63349 (F E W.), JHU-UMD Diabetes Research Center, P60DK079637 (F E W), and an award to L H from Johns Hopkins Institute for Clinical and Translational Research funded by the National Center for Advancing Translational Sciences, UL1TR000424-06.

\section{References}

Aguilar D, Chan W, Bozkurt B, Ramasubbu K \& Deswal A 2011 Metformin use and mortality in ambulatory patients with diabetes and heart failure. Circulation. Heart Failure 4 53-58. (doi:10.1161/CIRCHEARTFAILURE.110.952556)

Bailey CJ \& Day C 1989 Traditional plant medicines as treatments for diabetes. Diabetes Care 12 553-564. (doi:10.2337/diacare.12.8.553)

Evans JM, Donnelly LA, Emslie-Smith AM, Alessi DR \& Morris AD 2005 Metformin and reduced risk of cancer in diabetic patients. BMJ 330 1304-1305. (doi:10.1136/bmj.38415.708634.F7)

Goodman RH \& Smolik S 2000 CBP/p300 in cell growth, transformation, and development. Genes and Development 14 1553-1577. (doi:10.1101/ gad.14.13.1553) http://joe.endocrinology-journals.org DOI: 10.1530/JOE-14-0084
(C) 2014 Society for Endocrinology Printed in Great Britain
Published by Bioscientifica Ltd 
Gundewar S, Calvert JW, Jha S, Toedt-Pingel I, Ji SY, Nunez D, Ramachandran A, Anaya-Cisneros M, Tian R \& Lefer DJ 2009 Activation of amp-activated protein kinase by metformin improves left ventricular function and survival in heart failure. Circulation Research 104 403-411. (doi:10.1161/CIRCRESAHA.108.190918)

Guo S 2014 Insulin signaling, resistance, and the metabolic syndrome: insights from mouse models to disease mechanisms. Journal of Endocrinology 220 T1-T23. (doi:10.1530/JOE-13-0327)

He L, Sabet A, Djedjos S, Miller R, Sun X, Hussain MA, Radovick S \& Wondisford FE 2009 Metformin and insulin suppress hepatic gluconeogenesis through phosphorylation of CREB binding protein. Cell 137 635-646. (doi:10.1016/j.cell.2009.03.016)

He L, Naik K, Meng S, Cao J, Sidhaye AR, Ma A, Radovick S \& Wondisford FE 2012 Transcriptional co-activator p300 maintains basal hepatic gluconeogenesis. Journal of Biological Chemistry 287 32069-32077. (doi:10.1074/jbc.M112.385864)

He L, Cao J, Meng S, Ma A, Radovick S \& Wondisford FE 2013 Activation of basal gluconeogenesis by co-activator p300 maintains hepatic glycogen storage. Molecular Endocrinology 27 1322-1332. (doi:10.1210/ me.2012-1413)

Hundal RS, Petersen KF, Mayerson AB, Randhawa PS, Inzucchi S, Shoelson SE \& Shulman GI 2002 Mechanism by which high-dose aspirin improves glucose metabolism in type 2 diabetes. Journal of Clinical Investigation 109 1321-1326. (doi:10.1172/JCI0214955)

Landman GW, Kleefstra N, van Hateren KJ, Groenier KH, Gans RO \& Bilo HJ 2010 Metformin associated with lower cancer mortality in type 2 diabetes: ZODIAC-16. Diabetes Care 33 322-326. (doi:10.2337/ dc09-1380)

Libby G, Donnelly LA, Donnan PT, Alessi DR, Morris AD \& Evans JM 2009 New users of metformin are at low risk of incident cancer: a cohort study among people with type 2 diabetes. Diabetes Care 32 1620-1625. (doi:10.2337/dc08-2175)

Liu Y, Dentin R, Chen D, Hedrick S, Ravnskjaer K, Schenk S, Milne J, Meyers DJ, Cole P, Yates J III et al. 2008 A fasting inducible switch modulates gluconeogenesis via activator/coactivator exchange. Nature 456 269-273. (doi:10.1038/nature07349)

Nadeau KJ, Ehlers LB, Zeitler PS \& Love-Osborne K 2009 Treatment of non-alcoholic fatty liver disease with metformin versus lifestyle intervention in insulin-resistant adolescents. Pediatric Diabetes 10 5-13. (doi:10.1111/j.1399-5448.2008.00450.x)

Roussel R, Travert F, Pasquet B, Wilson PW, Smith SC Jr, Goto S, Ravaud P, Marre M, Porath A, Bhatt DL et al. 2010 Metformin use and mortality among patients with diabetes and atherothrombosis. Archives of Internal Medicine 170 1892-1899. (doi:10.1001/archinternmed.2010.409)

Schwimmer JB, Behling C, Newbury R, Deutsch R, Nievergelt C, Schork NJ \& Lavine JE 2005 Histopathology of pediatric nonalcoholic fatty liver disease. Hepatology 42 641-649. (doi:10.1002/hep.20842)

Shaw RJ, Lamia KA, Vasquez D, Koo SH, Bardeesy N, Depinho RA, Montminy M \& Cantley LC 2005 The kinase LKB1 mediates glucose homeostasis in liver and therapeutic effects of metformin. Science $\mathbf{3 1 0}$ 1642-1646. (doi:10.1126/science.1120781)

Takashima M, Ogawa W, Hayashi K, Inoue H, Kinoshita S, Okamoto Y, Sakaue H, Wataoka Y, Emi A, Senga Y et al. 2010 Role of KLF15 in regulation of hepatic gluconeogenesis and metformin action. Diabetes 59 1608-1615. (doi:10.2337/db09-1679)

Wilcock C \& Bailey CJ 1994 Accumulation of metformin by tissues of the normal and diabetic mouse. Xenobiotica 24 49-57. (doi:10.3109/ 00498259409043220)

Witters LA 2001 The blooming of the French lilac. Journal of Clinical Investigation 108 1105-1107. (doi:10.1172/JCI14178)

Zhou G, Myers R, Li Y, Chen Y, Shen X, Fenyk-Melody J, Wu M, Ventre J, Doebber T, Fujii N et al. 2001 Role of AMP-activated protein kinase in mechanism of metformin action. Journal of Clinical Investigation $\mathbf{1 0 8}$ 1167-1174. (doi:10.1172/JCI13505)

Zhou XY, Shibusawa N, Naik K, Porras D, Temple K, Ou H, Kaihara K, Roe MW, Brady MJ \& Wondisford FE 2004 Insulin regulation of hepatic gluconeogenesis through phosphorylation of CREB-binding protein. Nature Medicine 10 633-637. (doi:10.1038/nm1050)

Received in final form 8 March 2014

Accepted 17 March 2014

Accepted Preprint published online 17 March 2014
(C) 2014 Society for Endocrinology Printed in Great Britain 